\title{
Immune Regulation of "Nourishing Yin and Qi, Activating Blood Circulation and Detoxifying" in Elderly Diabetic Patients Complicated with Coronary Heart Disease
}

\author{
Fu Xianzhao*, Huang Guang Ming, Qiu Hai Xian, Li Chunyan, Huang Wenhua, Cao Qiuxia, \\ Lu Mian
}

The Affiliated Hospital of Youjiang Medical University for Nationalities, Baise, China

Email address:

1620100638@qq.com (Fu Xianzhao)

${ }^{*}$ Corresponding author

To cite this article:

Fu Xianzhao, Huang Guang Ming, Qiu Hai Xian, Li Chunyan, Huang Wenhua, Cao Qiuxia, Lu Mian. Immune Regulation of "Nourishing Yin and Qi, Activating Blood Circulation and Detoxifying" in Elderly Diabetic Patients Complicated with Coronary Heart Disease. Science Journal of Public Health. Vol. 8, No. 6, 2020, pp. 161-167. doi: 10.11648/j.sjph.20200806.12

Received: September 19, 2020; Accepted: October 26, 2020; Published: November 4, 2020

\begin{abstract}
Objective: To investigate the immune regulation of "nourishing Yin and Qi, activating blood circulation and detoxifying" in elderly diabetic patients complicated with acute coronary syndrome (ACS), and analyze its possible mechanisms. Methods: 69 diabetic patients aged 50-75 years and complicated with ACS, from March 2019 to February 2020 in the Department of Cardiology and Traditional Chinese medicine (TCM) in the Affiliated Hospital of Youjiang Medical University for Nationalities were randomly divided into control group (34 cases) and treatment group (35 cases). The control group was given western medicine standardized treatment, while the treatment group were treated with HJJR on the basis of routine Western medical treatment, and the therapeutic course for all were 2 months. Before and after treating, in the two groups, the levels of pro-inflammatory factors [C-reactive protein (CRP), interleukin-8 (IL-8), tumor necrosis factor - $\alpha$ (TNF $\alpha$ ) and anti-inflammatory factors [IL-10, adiponectin (ADPN)] were detected. The clinical symptoms of chest tightness, chest pain, attacking frequency and duration, palpitation, shortness of breath, fatigue and the total scores of syndromes in the two groups were detected. At the same time, electrocardiogram (ECG) changes and adverse reactions were observed. Results: Compared with before treatment, after treating, the levels of pro-inflammatory factors CRP, IL-8, TNF - $\alpha$ in the two groups were evidently decreased, while the anti-inflammatory factors IL-10 and ADPN were obviously increased, but the changes in the treatment group were more significant (all $\mathrm{P}<0.05$ ); the scores of frequency chest tightness and chest pain in both groups were decreased, while the duration of chest tightness and chest pain, fatigue and total score of TCM syndromes in the treatment group were significantly lower than those in the control group (all $\mathrm{P}<0.05$ ); the number of ST down segment, T-wave flattening, T-wave inversion in ECG were significantly lower than before treatment, with more obviously increased in the treatment group, but no statistically significant. No other adverse reactions were found in the two groups during the medication. Conclusion: HJJR can significantly reduce the levels of CRP, IL-8, TNF - $\alpha$, and increase the levels of IL-10 and ADPN in the serum of diabetic elderly patients complicated with ACS, suggesting that the effect of "nourishing yin and Qi, activating blood circulation and detoxifying" can regulate the balance mechanism of pro-inflammatory and anti-inflammatory factors, and bring into the protective effect on elderly diabetic patients with ACS.
\end{abstract}

Keywords: Pro-inflammatory Cytokines, Anti-inflammatory Cytokines, Elderly Diabetes, Acute Coronary Syndrome, Huoxue Jiedu Jiangtang Recipe

\section{Introduction}

Atherosclerosis (AS) and type 2 diabetes are both chronic low-grade inflammatory diseases. In recent years, studies have shown that the activation of the immune system, the inflammatory cell infiltration and the release of inflammatory mediators play the important roles in the occurrence and development of coronary heart disease (CHD) [1-2]. 
Inflammation is a major cause of cardiovascular events in the development of arteriosclerosis, and the imbalance of pro-inflammatory and anti- inflammatory factors plays the significant role in the pathogenesis of CHD [3]. Research shows that pathological immune response is one of the pathogenesis of diabetes, and the abnormal immune regulation plays an critical factor in the occurrence and development of type 2 diabetes [4]. Insulin resistance and the relative deficiency of insulin function are the activating factors of natural immunity in type 2 diabetic patients, and produce high levels of cytokinemia, which, as mediators and regulators of immune response, play key elements in the development of atherosclerotic plaque [5]. The immune function in elderly diabetic CHD is lower than that of young and middle-aged patients, therefore, by correcting the immune disorder, adjusting the number and proportion of lymphocyte subsets, maintaining the normal function of the immune system, adjusting the balance of promoting inflammation and anti-inflammatory, it will be possible to improve the elderly diabetic CHD more significantly than that of young patients [5-6]. According to traditional Chinese medicine (TCM), diabetes mellitus and its complicated AS are based on the basic pathogenesis of Qi-Yin deficiency coexisting with dry-heat accumulation, which can lead to blood circulating slowly and stasis, produce phlegm, and with the passing of time, accumulation of heat, phlegm, blood stasis can turn into toxins, forming plaques (that is, atherosclerotic plaques) [7]. In this study, by ameliorating the balance regulation in pro-inflammatory and anti-inflammatory factors in diabetic patients with acute coronary syndrome (ACS), the Huoxue Jiedu Jiangtang Recipe (HJJR), with the functions of "nourishing yin and Qi, activating blood circulation and detoxifying", softening and eliminating accumulation, were explored, and the complementary advantages of TCM and Western medicine will be improved to enhance the clinical prevention and treatment for diabetic ACS.

\section{Materials and Methods}

\subsection{Diagnostic Criteria}

\subsubsection{Diagnostic Criteria of Western Medicine}

ACS was diagnosed according to the " Unstable angina and non-ST-elevation myocardial infarction guidelines for diagnosis and treatment" (2007 Edition) [8], and 2019 Chinese Society of Cardiology (CSC) guidelines for the diagnosis and management of patients with ST-segment elevation myocardial infarction [9], published by cardiovascular branch of Chinese Medical Association and editorial board of Chinese cardiovascular disease Journal. Diabetes meets the diagnostic criteria of Chinese guidelines for the prevention and treatment of type 2 diabetes (2017 Edition) [10].

\subsubsection{TCM Discriminate Standard}

The diagnosis criteria for Qi-Yin deficiency and phlegm-blood stasis type in diabetic chest obstruction, refer to "the new guiding principles of Chinese medicine for clinical research" [11].

\subsection{Inclusion Criteria}

The diabetic ACS patients who failed to undergo PCI for various reasons, with Qi-Yin deficiency and phlegm blood stasis syndrome, aged between 50 and 75 years old, and who were approved by the hospital ethics committee, agreed and signed the informed consent.

\subsection{Exclusion Criteria}

Non-diabetic patients; patients with no both Qi and Yin deficiency, no phlegm and blood stasis block; patients with severe heart valve disease, cardiomyopathy, heart failure (NYHA grade IV); patients with severe liver failure and chronic renal insufficiency; ACS patients undergoing revascularization.

\subsection{Rejection and Shedding Criteria}

Patients who quit voluntarily and do not want to continue to cooperate with treatment, who lose follow-up or with poor compliance; Patients with incomplete data affecting the judgment of curative effect and safety; Patients whose condition worsens due to other reasons and were in danger of life.

\subsection{Clinical Data}

The 69 cases were all from the inpatients in the Department of Cardiology and TCM in the Affiliated Hospital of Youjiang Medical University for Nationalities from March 2019 to February 2020, and met the inclusion criteria. There were no significant differences in gender, age, duration of diabetes mellitus, duration of coronary heart disease, hypertension, diagnostic constituent ratio, and cardiac function grade between the two groups $(P>0.05)$, and were comparable (The comparison of clinical data were shown in Table 1).

Table 1. The comparison of clinical data.

\begin{tabular}{llllllll}
\hline group & n & male & female & Age & DM (years) & CHD (months) & HTN (cases, \%) \\
\hline Control group & 34 & 19 & 15 & $66.31 \pm 13.11$ & $11.77 \pm 5.51$ & $37.12 \pm 14.66$ \\
Treatment group & 35 & 20 & 15 & $67.45 \pm 12.22$ & $12.91 \pm 5.95$ & $36.26 \pm 15.94$ & $15(31.03)$ \\
\hline
\end{tabular}

\begin{tabular}{llll}
\hline \multirow{2}{*}{ group } & \multicolumn{4}{c}{ Diagnosis constitution [cases (\%)] } \\
\cline { 2 - 4 } & UAP & STEMI & NSTEMI \\
\hline Control group & $17(50.00)$ & $8(23.52)$ & $9(26.47)$ \\
Treatment group & $18(51.42)$ & $9(25.71)$ & $8(22.85)$ \\
\hline
\end{tabular}

Notes: DM (diabetes mellitus), CHD (coronary heart disease), HTN (Hypertension), UAP (Unstable angina pectoris), STEAMI (ST-elevation myocardial infarction), NSTEAMI (Non-ST-elevation myocardial infarction), NYHA (New York Heart Association). 


\subsection{Therapeutic Method}

All cases were randomly assigned to control group (34 cases) treated with standardized western medicine, and treatment group (35 cases) treated with HJJR on the basis of standardized treatment, which specifically involved: the treatment of diabetes mellitus according to the Chinese guidelines for the prevention and treatment of type 2 diabetes [10], cardiovascular drugs consulting the guidelines for diagnosis and treatment of acute coronary syndrome [8-9], including anti-platelet therapy (Aspirin: the first loading dose was $300 \mathrm{mg}$ chewed, and the maintenance dose was 75-100 mg orally; clopidogrel: the first loading dose was $300 \mathrm{mg}$ oral, and the maintenance dose was $75 \mathrm{mg}$ ), anticoagulant therapy (Low-molecular-weight Heparin Sodium was injected subcutaneously twice a day for 2-8 days), ACEI (If there is taboo, choose ARB), Beta blocker (for patients without contraindications, adjust dosage according to blood pressure, heart rate and cardiac function), Lipid regulating drugs (simvastatin 20mg, taken After dinner, before going to bed), Nitrates (isosorbide nitrate $10 \mathrm{mg}$, twice daily). HJJR, which composed of Ginseng $10 \mathrm{~g}$, Astragalus $15 \mathrm{~g}$, Ophiopogon $15 \mathrm{~g}$, Schisandra $10 \mathrm{~g}$, Cornus officinalis $10 \mathrm{~g}$, Rehmannia $15 \mathrm{~g}$, yam $15 \mathrm{~g}$, rhubarb $5 \mathrm{~g}$, turtle shell $15 \mathrm{~g}$, peach kernel $10 \mathrm{~g}$, cortex moutan $10 \mathrm{~g}$, Salvia miltiorrhiza 10 $\mathrm{g}$, Coptis $8 \mathrm{~g}$, were decocted in water for about $300 \mathrm{ml}$, taken three times a day (morning, middle and evening). During the treatment, it is forbidden to use other traditional Chinese medicine for tonifying Qi and nourishing Yin, promoting blood circulation and removing blood stasis. The treating course was two months, during which, appropriate treatment could be given to treat other concomitant diseases, such as anti infective therapy for combined infection, on the premise of not affecting the efficacy of the trial drug.

\subsection{Observation Index and Detection Method}

\subsubsection{TCM Clinical Syndrome Score (Refer to "the New Guiding Principles of Chinese Medicine for Clinical Research") [11].}

(1) Chest pain

No chest pain was scored 0; Chest pain attacking 2-6 times a week, lasting less than 5 minutes each time, taking 1-4 tablets of nitroglycerin per week, 2 scores; Chest pain attacking 1-3 times a day, lasting less than 6-9 minutes each time, taking 5-9 tablets of nitroglycerin per week, 4 scores; Chest pain attacking more than 4 times a day, lasting less than 10 minutes each time, taking less than 10 tablets of nitroglycerin per week, 6 scores.

(2) Chest tightness

No chest tightness was scored 0; Occasionally feel chest tightness, and can be relieved by rest, 2 scores; Chest tightness occurs more frequently, but patients can live and work normally, 4 points; Chest tightness continued to puzzle, affecting patients normal life and working, 6 points.

(3) Palpitation

No palpitation was scored 0 ; Occasional palpitation can be relieved by itself, 1 score; palpitation attacks frequently, but patients can persist in work, 2 scores; palpitations continue to occur, can not be relieved, and even affect normal life and work, 3 scores.

(4) Shortness of breath

No shortness of breath, 0 score; Shortness of breath appears after the activity, 1 scores; Slight activity produces shortness of breath, 2 scores; Even if not active, patient also developed shortness of breath, 3 scores.

(5) Fatigue

No fatigue was scored 0; Occasionally fatigue, but can be relieved by itself, 1 score; Often feel tired, but can persist in working, 2 scores; Fatigue continue to occur, can not be relieved, and even affect normal life and work, 3 scores.

\subsubsection{Electrocardiogram (Refer to "the New Guiding Principles of Chinese Medicine for Clinical Research") [11]}

The number of downward ST segments, flatting $\mathrm{T}$ waves and inversion $\mathrm{T}$ waves were observed before and after treatment in the two groups.

\subsubsection{Detection of Pro-inflammatory and Anti-inflammatory Factors}

On the day of admission and the morning of discharge, $5 \mathrm{ml}$ of fasting venous blood of the two groups were collected and placed in the anticoagulant tubes containing EDTA. After centrifugation $(3000 \mathrm{r} / \mathrm{min})$, the supernatant were collected and placed in $-80{ }^{\circ} \mathrm{C}$ refrigerator for frozen storage. The CRP, IL-8, TNF- $\alpha$, IL-10 and adiponectin (ADPN), whose kits were provided by Wuhan doctor de Bioengineering Co., Ltd. were determined by enzyme-linked immunosorbent assay (ELISA), which specific steps were carried out according to the instructions.

\subsection{Statistical Methods}

SPSS17.0 medical statistical software was used to analyze all the data. The measurement data were expressed as $\left(\bar{x}_{ \pm \mathrm{S}}\right)$, and the paired t-test was used to compare the data before and after clinical trials, while the t-test in two independent samples was used for the comparison between groups. The count data were expressed as $\%$ and tested by $x^{2} . P<0.05$ was statistically significant.

\section{Result}

\subsection{Comparison of Pro-inflammatory and Anti-inflammatory Factors Between the Two Groups Before and After Treatment (Table 2)}

Intra group comparison, compared with that before treatment, the levels of serum pro-inflammatory factors CRP, IL-8 and TNF- $\alpha$ in the two groups were significantly lower $(P<0.01$ or $P<0.05)$, while the anti-inflammatory factors IL-10 and ADPN were significantly increased $(P<0.01$ or $P<0.05)$; Comparison between groups, after treatment, the levels of CRP, IL- 8 and TNF- $\alpha$ in the treatment group were 
significantly lower than those in the control group $(\mathrm{P}<0.05)$, while the anti-inflammatory factors IL-10 and ADPN in the treatment group were significantly higher than those in the control group $(\mathrm{P}<0.05)$

Table 2. Comparison of pro-inflammatory and anti-inflammatory factors between the two groups before and after treatment ( $\bar{x} \pm s)$.

\begin{tabular}{|c|c|c|c|c|c|c|c|}
\hline \multirow{2}{*}{ Groups } & & \multirow{2}{*}{$\mathbf{n}$} & \multicolumn{3}{|c|}{ Pro-inflammatory factors } & \multicolumn{2}{|c|}{ Anti-inflammatory factors } \\
\hline & & & CRP (mg/L) & IL-8 (ng/L) & TNF- $\alpha(n g / L)$ & $\operatorname{ADPN}(\mathrm{mg} / \mathrm{L})$ & IL-10 (ng/L) \\
\hline \multirow{2}{*}{ Control group } & Before treatment & 34 & $4.11 \pm 0.39$ & $158.38 \pm 31.44$ & $45.64 \pm 11.87$ & $8.15 \pm 2.48$ & $31.94 \pm 13.12$ \\
\hline & After treatment & 34 & $3.33 \pm 0.48^{\Delta}$ & $142.31 \pm 34.56^{\Delta}$ & $36.02 \pm 13.11^{\Delta}$ & $12.14 \pm 3.78^{\Delta}$ & $44.13 \pm 10.14^{\Delta}$ \\
\hline \multirow{2}{*}{ Treatment group } & Before treatment & 35 & $4.22 \pm 0.44$ & $166.61 \pm 31.86$ & $46.10 \pm 12.12$ & $8.32 \pm 2.28$ & $30.89 \pm 11.15$ \\
\hline & After treatment & 35 & $2.92 \pm 0.36^{\mathbf{\Delta}}$ & $125.28 \pm 27.28^{\mathbf{4}}$ & $26.22 \pm 10.81^{\mathbf{\Delta}}$ & $14.87 \pm 3.13^{\mathbf{*}}$ & $46.46 \pm 10.17^{\mathbf{\Delta}}$ \\
\hline
\end{tabular}

Note: compared with before treatment, $\Delta P<0.05, \mathbf{\Delta} P<0.01$; compared with the control group, $* P<0.05$.

\subsection{Comparison of ECG Results Between the Two Groups Before And after Treatment (Table 3)}

Intra group comparison, compared with that before treatment, the number ST falling, $\mathrm{T}$ wave depression and inversion in ECG decreased more obviously in two groups

Table 3. Comparison of ECG between the two groups before and after treatment (number, $\bar{x}_{ \pm s}$ ).

\begin{tabular}{|c|c|c|c|c|c|}
\hline Groups & & $\mathbf{n}$ & ST moving down & T-wave flatting & T-wave inversion \\
\hline \multirow{2}{*}{ Control group } & Before treatment & 34 & $4.7 \pm 0.7$ & $2.6 \pm 0.7$ & $2.4 \pm 1.1$ \\
\hline & After treatment & 34 & $3.2 \pm 0.6^{\Delta}$ & $2.3 \pm 0.6^{\Delta}$ & $2.1 \pm 0.8^{\Delta}$ \\
\hline \multirow{2}{*}{ Treatment group } & Before treatment & 35 & $4.8 \pm 0.7$ & $2.7 \pm 0.9$ & $2.5 \pm 1.4$ \\
\hline & After treatment & 35 & $2.9 \pm 0.5^{\Delta}$ & $2.2 \pm 0.8^{\Delta}$ & $2.0 \pm 0.7^{\Delta}$ \\
\hline
\end{tabular}

Note: compared with that before treatment, $\Delta \mathrm{P}<0.05$

\subsection{Comparison of TCM Clinical Syndrome Scores Between the Two Groups Before and After Treatment (Table 4)}

Compared with that before treatment, the duration and frequency of chest tightness and chest pain in the two groups
$(\mathrm{P}<0.05)$; Comparison between groups, after treatment, the number of ST falling, $\mathrm{T}$ wave depression and inversion in the treatment group were less than that in the control group, but the difference was not statistically significant $(\mathrm{P}>0.05)$.

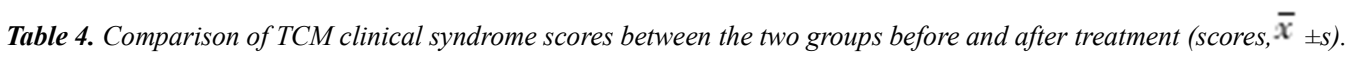

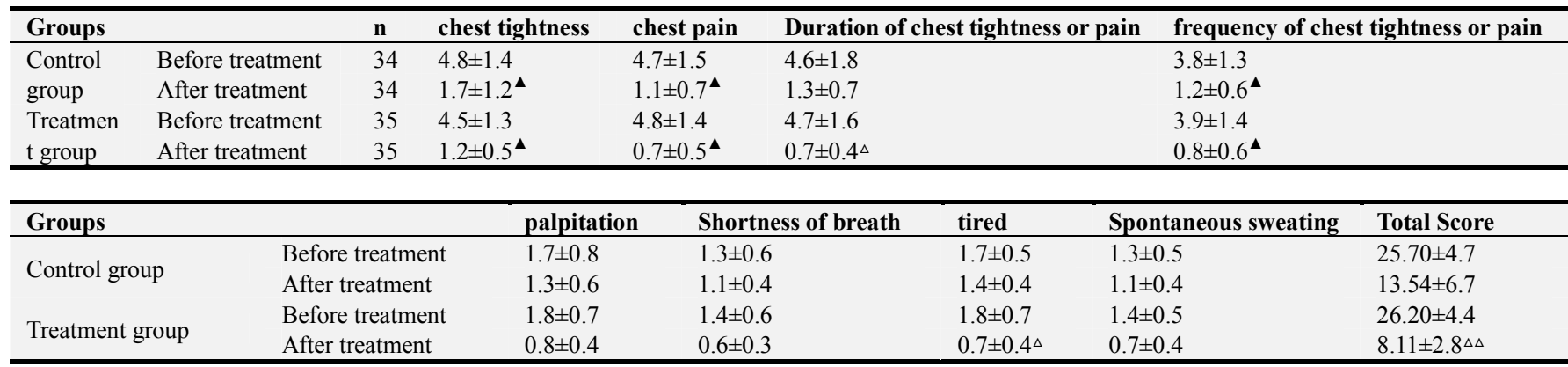

Note: compared with before treatment, $\Delta P<0.01$; compared with the control group, $\Delta P<0.05, \Delta \Delta P<0.01$.

\subsection{Adverse Reactions}

During the medication, the blood, urine, stool routine test, liver and kidney function test were not significantly abnormal, no other adverse reactions were found in two groups of patients

\section{Discussion}

Atherosclerosis (AS) is an inflammatory reaction, in which, inflammation and inflammatory factors play an important role in the occurrence and development of atherosclerotic plaque from from a steady state to a non-steady state [12]. By were decreased $(\mathrm{P}<0.01)$; compared with the control group, the duration of chest tightness and pain, fatigue and total score of syndromes in the treatment group were decreased $(\mathrm{P}<0.05, \mathrm{P}<0.01)$. 
mellitus is a vulnerable CHD patient, whose pathological mechanisms more complex than that of non diabetic person, involve vascular endothelial damage, excessive oxidative stress, inflammatory response, with metabolic memory function and multiplicative effect with other cardiovascular risk factors, more harmful to the heart [15-16]. Type 2 diabetes participates in the activation of nonspecific immune system, due to the disorder of glucose metabolism and accumulation of a large number of metabolites, which in turn aggravate the diabetic development, break down the dynamic balance between pro-inflammatory cytokines and anti-inflammatory cytokines, playing an important role in the occurrence and development of type 2 diabetes [4, 5]. At the same time, with the increase of age, the cellular immune function of the elderly diabetic patients have been damaged, with helper lymphocyte $\mathrm{T}$ cell $\left(\mathrm{CD}^{4+}\right)$ decreasing, while $\mathrm{CD}^{8+}$ of $\mathrm{T}$ lymphocytes with inhibition and killing ability increasing relatively, showing the number of $\mathrm{CD}^{4+} \mathrm{T}$ and the ratio of $\mathrm{CD}^{4+} \mathrm{T} / \mathrm{CD}^{8+} \mathrm{T}$ decreasing, the immune regulatory center disordering, leading to over activation of pro-inflammatory factors, insufficient expression of anti-inflammatory factors and the imbalance between pro-inflammatory and anti-inflammatory $[4,5]$.

Atherosclerosis is often caused by the imbalance between the mechanisms of arterial injury (promoting inflammation) and repairing protection (anti-inflammatory), which can produce destructive inflammatory effect and intensify positive feedback result, aggravate arterial injury and promote the occurrence and development of AS, while the balance of inflammatory response is beneficial to the arterial wall repairing [17]. The pro-inflammatory factors can promote AS formation by damaging the endothelium, inducing the monocyte adhesion to endothelium, and migrating to the subintima, moreover, pro-inflammatory factors can also increase plaque instability by promoting extracellular matrix degradation and inhibiting its repair [18]. CRP can induce the production of tissue factor and monocyte, and trigger the process of coagulation, besides, as a chemokine of fibrinogen, CRP can make macrophages adhere to the surface of endothelium and then transplant to intima [19]. IL-8, which can be used as an indicator to predict the early pathological changes of ACS, and which produce in vascular endothelial cells, smooth muscle cells and monocytes, can activate inflammatory reaction and induce tissue damage, and is positively correlated with the risk of serious coronary heart disease [17]. TNF $-\alpha$ is related to the stability of coronary plaques, which can directly damage vascular endothelial cells, cause inflammatory pathological damage, activate macrophages in atherosclerotic plaque to secrete matrix metalloproteinases (MMPs) and destroy the stability of plaques [20].

A balance or ratio between pro-inflammatory and anti-inflammatory forces can more comprehensively reflect the inflammatory response of the body, regulate the occurrence and development of acute coronary syndrome of coronary heart disease. Researches showed that IL-10, as an anti-inflammatory factor, can stabilize AS plaques by inhibiting monocyte adhesion, checking vascular smooth muscle cell proliferation, antagonizing the inflammatory activity of pro-inflammatory factors and down regulating the inflammatory response [21]. However, when acute coronary syndrome occurs (ACS), although IL-10 is activated by inflammation, it is not able to antagonize the inflammatory damage due to its imbalance with the degree of inflammation [21]. Adiponectin, as another inhibitor of inflammation, can inhibit the uptake of cholesterol ester by macrophages, reduce the expression of scavenger receptor gene in macrophages, inhibit the macrophages transforming into foam cells, reduce monocytes secreting TNF- $\alpha$ and inhibit its mediating inflammatory response, and significantly low adiponectin expression level in patients with coronary heart disease, will directly affect the AS occurrence and development [18].

In the elderly diabetic patients, the immune function decrease in parallel with the aging of the body, and with the cellular and humoral immune functions decreasing [2, 5], therefore, immunomodulatory therapy can provide a new direction for the adjustment and improvement of treatment strategy for diabetic CHD, which is the demand of prevention and treatment of senile diabetic CHD.

According to TCM theory, diabetes mellitus and coronary heart disease coexist in the development and evolution on the basis of Yin-deficiency and Dry-heat-accumulation in Xiao $\mathrm{Ke}$, whose pathogenesis involves the following aspects:(1) Yin deficiency and dryness-heat can refine and turn the liquid into phlegm, which in turn can combine with blood stasis, blocking heart vessels and causing chest obstruction;(2) Yin deficiency and dryness-heat cause Qi-Yin insufficiency, and Qi incapability can do nothing for blood circulation, then blood stagnate and block the heart vascular, making chest pain;(3) The long onset of Qi-Yin insufficiency can evolved into Yin-Yang deficiency, and heart vessel block is easily caused by deficiency of heart Yang and the accumulation of cold pathogen (Arising from Yang Deficiency) [22]. The long-term over accumulation of dry-heat, phlegm dampness, blood stasis, can eventually turn into toxins [22]. The pathogenesis of diabetic coronary heart disease (CHD) can be summed up as "Qi-Yin deficiency, blood stasis and toxin accumulation" [7]. Therefore, the "Supplementing Qi and nourishing Yin, activating blood circulation and detoxifying" therapy is suitable for diabetic ACS [7]. Huoxue Jiedu Jiangtang formulation (HJJR) consist of ginseng, turtle shell, ophiopogon japonicus, astragalus, fructus corni, rhubarb, radix rehmanniae, rhizoma coptidis, salvia miltiorrhiza, cortex moutan, peach kernel, yam and fructus schisandrae chinensis, in which, ginseng, ophiopogon root, schisandra possess efficency of nourishing Yin, tonifying Qi, generating fluid, and nourishing heart; cornus, yam and rehmannia play the roles of nourishing kidney, filling Yin and suppressing Yang, for treating true Yin deficiency; turtle shell, rhubarb, cortex moutan and peach kernel are Eastern Han Dynasty medical expert Zhang Zhongjing's "Turtle shell pills" ingredients for treating "Accumulation", and turtle shell can nourish kidney Yin, clear away asthenia heat, soften and 
dissipate stagnation; salvia miltiorrhiza coordinated with peach kernel and cortex moutan, can promote blood circulation and make blood stasisr dissipated; hizoma coptidis combined with rhubarb can eliminate heat-toxin and dispell dampness; Astragalus membranaceus cooperated with ginseng, can invigorate Qi and generate blood, making toxins floated and dissipated. All prescriptions share the effects of "Nourishing Yin, Supplementing Qi, Activating circulation, Eliminating toxin, Softening plaques and Dissipating stasis", supplementing and attacking in combination [22].

\section{Conclusion}

In this study, it was found that the combination of HJJR and Western Medicine on the basis of the routine treatment in the elderly diabetic patients with ACS, can significantly reduce the levels of pro-inflammatory factors CRP, IL-8, TNF- $\alpha$, enhance the expression of anti-inflammatory factors IL-10, adiponectin, regulate the anti-inflammatory/ pro-inflammatory balance, effectively inhibit the inflammatory response, improve the myocardial blood supply (The numbers of ST segment depression, T-wave flattening or inversion were all decreased, suggesting ischemic condition improved), relieve the TCM clinical symptoms of chest pain, chest distress, palpitation, asthenia, shortness of breath and so on. This shows that, by regulating the balance of pro-inflammatory and anti-inflammatory, HJJR inhibit harmful inflammatory factors, enhance the effect of beneficial cytokines, achieve the therapeutic effect on elderly diabetic ACS, which provide a theoretical basis for clinical application of "nourishing yin and Qi, promoting blood circulation and detoxification" in the treatment of elderly diabetic coronary heart disease. Considering the small sample size, there are inevitably some shortcomings. In the future clinical work, multi center, large sample and prospective clinical comparative studies should continue to seek complementary advantages of traditional Chinese and Western medicine, and reduce the incidence and mortality in elderly patients with diabetic CHD.

\section{Acknowledgements}

This research is supported by National Natural Science Foundation of China (81960833); Guangxi emphasis research and development program (2017AB45042); Guangxi Natural Science Foundation (2019JJA140146).

\section{References}

[1] CHEN Yuanyuan, CHEN Yafen, SUN Xueran, et al. Regulation mechanism of AGEs on FSTL1 expression in diabetes with atherosclerosis. International Journal of Cardiovascular Disease, 2019, 46 (1): 31-35.

[2] Itariu B K, Stulnig T M. Autoimmune aspects of type 2 diabetes mellitus a mini-review [J]. Gerontology, 2014, 60: 189-196.
[3] ZHONG Qiaoqing, ZHU Lingyan, ZHANG Guogang, et al. Atherosclerotic cardiovascular disease in diabetes--mechanisms, clinical represent and management. Chinese Journal of Cardiovascular Review, 2018, 16 (12): 1069-1072.

[4] Donath MY, Shoelson SE. Type 2 diabetes as an inflammatory disease. Nat Rev Immunol, 2011, 11: 98-107.

[5] ZHU Yun-qing, HU Yun, MAO Xiao-ming. The research progress of immune disorders in Type 2 diabetes mellitus. Chinese Journal of Diabetes, 2015, 23 (9): 859-861.

[6] Zhu Dalong; $\mathrm{Mu}$ Yiming. Expert consensus on glucose-lowering pharmacotherapies in Chinese adults with type 2 diabetes and cardiovascular disease or chronic kidney disease. Chinese Journal of Endocrinology and Metabolism, 2020, 6: 458-468.

[7] FU Xian-zhao, XU Jing, HUANG Wen-hua, et al. Strategy of Stasis-resolving and Detoxification on Diabetic Coronary Heart Disease [J]. JETCM, 2014, 23 (11): 2024-2027.

[8] Cardiovascular branch of Chinese Medical Association, Editorial board of Chinese Journal of cardiovascular disease. Unstable angina and non-ST-elevation myocardial infarction guidelines for diagnosis and treatment. Chin J Cardiol, 2007, 35 (4): 295-304.

[9] Cardiovascular branch of Chinese Medical Association, Editorial board of Chinese Journal of cardiovascular disease. 2019 Chinese Society of Cardiology (CSC) guidelines for the diagnosis and management of patients with ST-segment elevation myocardial infarction. Chin J Cardiol, 2019, 47 (10): 766-783.

[10] Diabetes branch of Chinese Medical Association. Guidelines for the prevention and treatment of type 2 diabetes in China. Chinese Journal of diabetes. 2018, 10 (1): 4-65.

[11] Zheng Xiaoyi. the new guiding principles of Chinese medicine for clinical research. BeiJing: China Medical Science and Technology Publishing House, 2002: 68-73.

[12] Shen Ying, Ding Fenghua, Lu Lin, Zhang Ruiyan, Shen Weifeng. Blood pressure management in diabetic patients with coronary artery disease. Chinese Journal of Cardiology, 2020, 1: 5-9.

[13] Wan Chunling, Yang Yaru, Jiao Yahui. Clinical analysis of adverse cardiovascular events in patients with acute coronary syndrome complicated with type 2 diabetes mellitus. J Clin Psychosom Dis, 2018, 24 (4): 20-22.

[14] Hong tianpei, Mu yiming, Zhu, dalong. Intervention for adults with pre-diabetes: A Chinese expert consensus. Chinese Journal of Endocrinology and Metabolism, 2020, 5: 371-380.

[15] XIAO Jian-yong, ZHANG He-nan, CAO Lu. An analysis of relationship between the severity of coronary artery lesion and risk factors of cardiovascular events in Tianjin. Chinese Critical Care Medicine, 2013, 25 (11): 650-654.

[16] PENG Xiao-ren, ZHAO Yan-fang, ZOU Da-jin. The role of diabetes mellitus as a risk factor of acute myocardial infarction. Chinese Critical Care Medicine, 2011, 23 (6): 322-327.

[17] CZHANG Zengping, WANG Weijia, KONG Yina, WANG Congrui. orrelation between the stability of carotid artherosclerotic plaque and the levels of serum adopinectin and IL-8 in patients with acute cerebral infraction. J Xuzhou Med Univ 2, 2019, 39 (4): 289-292. 
[18] $\mathrm{Hu} \mathrm{H}$, Wang $\mathrm{C}$, Jin $\mathrm{Y}$, et al. Catalpol Inhibits Homocysteine-induced Oxidation and Inflammation via Inhibiting Nox4/NF-kB and GRP78/PERK Pathways in Human Aorta Endothelial Cells. Inflammation. 2019 Feb; 42 (1): 64-80. doi: 10.1007/s10753-018-0873-9.

[19] Wang Ying, Wang Aihua, Tian Tian. Correlation of endothelin-1, adiponectin, C-reactive protein and related metabolic indexes in type 2 diabetes mellitus with atherosclerosis. Guangdong Medical Journal, 2019, 40 (7): 1020-1024.

[20] DANG Jingyi, HAO Qimeng, JIANG Na, et al. Relationship between serum lipoprotein (a), $\beta 2$-microglobulin and tumor necrosis factor- $\alpha$ levels and degree of coronary artery lesion in patients with acute coronary syndrome. Chin J Arterioscler, 2020, 28 (8): 702-706.

[21] SUN Jun, ZHENG Jiwei, JIA Fan. The relationship between serum IL-6 level of patients with type 2 diabetic mellitus and carotid atherosclerosis. Inr J Lab Med, 2018, 39 (5): 548-554.

[22] Fu Xianzhao, Huang Zhenfeng, Huang Wenhua, et al. Review and Prospect of the Preventive Effect of "Supplementing Qi and Nourishing Yin, Activating Blood Circulation and Detoxifying" on Diabetes Mellitus Complicated with Acute Coronary Syndrome. [J]. Chin J TCM WM Crit Care, 2017, 24 (5): 547-551. 